\title{
Líneas de políticas y estrategias de educación al trabajar con jóvenes universitarios en situación de diferencia y desigualdad
}

Policy guidelines and educational strategies when working with university youths under conditions of disparity and inequality

\author{
Marta Ofelia Chaile \\ Consejo de Investigación de la \\ Universidad Nacional de Salta \\ Argentina \\ chailemo@gmail.com
}

\section{Resumen}

En el trabajo se trazan líneas de políticas y estrategias de un proceso educativo, a modo de propuesta de desarrollo en una universidad cuyos estudiantes mayoritariamente proceden de contextos de desigualdad social y de diversidad cultural, lo que obra de modo desfavorecedor para el aprendizaje.

Se parte de referenciar las condiciones de carencias en el medio local, recurriendo a estudios e informes de investigación. Ello a su vez articula con la configuración de la matriz de los estudiantes ingresantes a los profesorados en ciencias, observándose sus limitaciones en la comprensión e interpretación cultural simbólica. Se manejan los conceptos de alfabetización académica y de habilitación política como metas de logro.

El trabajo avanza integrando aportes conceptuales de la antropología cultural y de la justicia distributiva, con los cuales se fundamenta la necesidad de revisar criterios de la tarea pedagógica en la universidad.

Palabras clave: políticas y estrategias educativas, desigualdad social, diferencia cultural, habilitación política, aula universitaria.

\begin{abstract}
In the work, lines of policies and strategies are drawn from an educational process, as a development proposal in a university whose students mostly come from contexts of social inequality and cultural diversity, which works in a way that is unfavorable for learning.
\end{abstract}


Reference is made to the lack of conditions in the local environment, using studies and research reports. This in turn articulates with the matrix's configuration of students entering to the science teacher's careers, observing its limitations in the understanding and cultural symbolic interpretation. The concepts of academic literacy and political empowerment are managed as goals of achievement.

The work advances integrating conceptual contributions of cultural anthropology and distributive justice, with which the need to revise criteria of the pedagogical task in the university is based.

Finally, an educational and cultural policy of improvement is promoted.

Key words: educational policies and strategies, social inequality, cultural difference, political empowerment, university classroom.

\section{Presentación}

En el presente trabajo se plantea el comprometido proceso de reflexionar, elaborar, construir alternativas viables y habilitantes de condiciones que favorezcan el desempeño competencial por parte de estudiantes universitarios, en el marco de una política para su promoción educativo-cultural.

Los aludidos estudiantes se inscriben -en el caso que aquí interesa- en los profesorados de ciencias exactas de una universidad pública y gratuita, para lo cual satisfacen el requisito de presentación del certificado de finalización de estudios de nivel Medio (o Secundario). Luego de la inscripción concurren a un curso de ingreso no obligatorio de seis semanas de duración. $\mathrm{Si}$ bien el ingreso es irrestricto, y el tal sentido se ofrece la oportunidad democrática del acceso sin limitaciones, en la práctica se observa que llega a la universidad una población estudiantil heterogénea.

Los hechos ocurren en una universidad pública localizada en el noroeste argentino. El desempeño de la tarea pedagógica se ubica en un contexto socio-económico-cultural limitado, con situación de bipolaridad social acusada, con bajo nivel de desarrollo laboral y económico, distante de la capital del país. A su vez la población se compone de familias descendientes de aquéllas instaladas en la época de la colonia española más un número significativo de pobladores de raíz étnica originaria.

El avance cultural favorece la promoción de los estudios universitarios y de este modo la concurrencia de sujetos procedentes de las familias de la provincia -y de la región- se cuenta entre los logros alcanzados en el proceso de expansión educativa.

No obstante, el entrecruzamiento de las condiciones referidas -contexto social de alcances modestos en lo económico, en lo ocupacional, en la participación cívica, y estudiantes de tales contextos que concurren a la universidad- estructura un escenario contradictorio. Ello 
porque si bien se habilita legalmente el ingreso irrestricto a los estudios de nivel superior, es relativo asegurar que los sujetos ingresantes demuestren condiciones pedagógicas logradas de modo óptimo durante los estudios cursados en los niveles previos del sistema educacional y en especial en el secundario. Hay un desfase de condiciones que acarrea problemas de baja calidad de los aprendizajes al ingresar a la universidad, que torna difícil sostener su ritmo de estudio, acompasado al nivel de exigencia pedagógica.

Aunque se logrado expandir democráticamente el acceso a la universidad de todos los sectores sociales, no todos de los estudiantes acceden con el potencial de capital cultural de base, requerido.

Hay un choque cultural en este acontecimiento del ingreso al profesorado universitario, que se observa desde las dificultades para manejar códigos lógicos y organizativos para la construcción de contenidos académicos en cuanto a su interpretación, significación y articulación cognoscitiva, al tiempo que faltan soportes actitudinales y hábitos que permitan actuar y sedimentar el proceso de ir armándose como sujeto universitario. La situación no deja afuera al docente del aula universitaria sino lo involucra ética y profesionalmente.

Pese a la renuencia cultural a instalarse, ubicarse y adaptarse al nuevo nivel del sistema educacional, la presencia de los nuevos sujetos reta o interpela porque el sistema se haga cargo de ellos, lo que se juzga válido cívica y pedagógicamente. En los últimos años se observa la acentuación de los déficits en lo educacional (Tenti Fanfani, 2003), sin poder dejar de aludir a las dificultades derivadas de la cuestión social y con ello a la ruptura y fragmentación del tejido social, articulado a la desocupación y al aumento de condiciones de pobreza o semipobreza de los hogares de la región.

Incide también una historia de carencias culturales acumuladas, en especial en los sectores sociales más humildes, lo que genera incredulidad en el potencial de los estudiantes de dicha procedencia, por parte del profesorado, que puede llegar a estigmatizar, diferenciar, predecir, descalificar su actuación.

Como en toda historia, los factores de poder se involucran y juegan a favor de intereses. La educación, como la cultura, se concentra o se distribuye. (cf., Cerletti, A., 2013; Gvirtz, S., Grinberg, S., Abregú, V. 2009). Y la educación, como puerta de la cultura (Bruner, 2000) habilita o inhabilita para la participación social.

\section{Un contexto social pauperizado}

La relación entre el contexto, sus condiciones y sus aportes o por el contrario su influencia contraproducente en la educación de los sujetos, se haya probada tanto mediante teorías y enfoques sociológicos (los reproductivistas críticos como Bourdieu, 1977; Bowles y Gintis, 1985; Baudelot y Establet, 1976); pero además se torna visible en la vida cotidiana de hogares y familias cuya pobreza social, cultural, económica, arrastra en caída descendente la calidad de vida de sus hijos. El entorno político y sus decisiones obran como motor de desarrollo o como ancla de hundimiento según la orientación que asuman, en particular en las sociedades latinoamericanas, con notable debilidad estructural de sus políticas y desarrollo social. 
En tal línea el norte argentino integra provincias que en el conjunto del país poco aportan al PBI nacional, siendo la cifra para Salta alrededor de 1 punto y medio $\left(11^{\circ}\right.$ en ranking. Igual a \$ 5.366 millones) en la década de inicios del 2000 luego de sufrir una profunda crisis económica, política y social:

Los datos oficiales, disponibles al año 2003, (INDEC) estiman el producto salteño en un $1.5 \%$ del PBI nacional. El sector servicios representa $60.1 \%$ de la producción de la provincia. El sector primario es relativamente importante $(22.3 \%)$ y el sector industrial participa relativamente poco en el total del producto (16.9\%). En relación a los datos regionales, Salta tiene un PBG per cápita apenas superior al promedio de la región NOA (Noroeste argentino) (Instituto de Estudios sobre Estado y Participación, 2008: 10).

Salta se ubica entre las provincias pobres del país, basando su economía en "actividades de baja complejidad tecnológica y de relativamente escaso valor agregado. Entre las actividades primarias se destacan el tabaco, poroto, azúcar y en los últimos años soja. Otras actividades primarias son la explotación de minerales de uso industrial (Salta es el primer productor de boratos) e hidrocarburos" (IDEP, 2008: 10).

Por su parte, utilizando como indicador la tasa de recuento de la pobreza, que señala el porcentaje de personas residentes en hogares pobres por insuficiencia de ingresos monetarios, el Instituto de Estudios Laborales y del Desarrollo Económico (2014) muestra la evolución de la pobreza en Salta durante la próxima pasada década. Para el último año y medio (2016, 2017) las cifras se han profundizado negativamente, considerando el cambio de rumbo de la política social, económica y laboral del nuevo gobierno en el país. Colque (2017) expresa en una entrevista el cuestionamiento al "rendimiento real del gasto público destinado a los sectores más humildes". Y agrega,

La tasa de pobreza ascendió del 29 por ciento a fines de 2015 al 32,9 por ciento en el tercer trimestre de 2016. Esto significaría un aumento de 1,5 millones de nuevos pobres, son cerca de 13 millones de personas por debajo de la línea de pobreza. Estos niveles serían los más altos de los registrados desde 2010. El Salta, históricamente, fueron superiores, por encima del 40 por ciento.

Relacionando desigualdad social y pobreza, Colque (2017) dice:

En la Argentina la desigualdad persistente y la pobreza estructural se debe a que desde hace más de 30 años se viene aplicando un modelo económico-productivo concentrado y desequilibrado, con efectos de exclusión y desigualdad a nivel sociolaboral. Es decir, que lo más preocupante no es en sí el nivel de pobres sino la desigualdad existente.

Esta relación entre pobreza y desigualdad también puede mirarse desde subcategorías analíticas de interpretación que hacen a la vulnerabilidad de los grupos sociales y sus formas de configurar factores como salud, vivienda, zona de radicación, acceso a transporte público, estado de rutas o caminos, acceso a servicios básicos, que el IELDE (2014) trabaja como multidimensionalidad del bienestar en tanto medida de los efectos de una pobreza estructural. 
La concentración de la actividad económica en pocas manos, la falta de fuentes laborales, con una ocupación intensiva en el empleo pública agrega causas que inciden en la configuración de una matriz de pobreza en la provincia, replicada con mayor alcance desde su capital hacia los departamentos del interior.

En esta presentación que pretende ubicar la realidad socio-económica de Salta, no se puede dejar de mencionar su pluralidad étnica, compartida con la vecina Jujuy. Los estudios del IDEP (2008) mencionan que, según el censo del 2001, hacen presencia efectiva los pueblos kolla (70505 habitantes), guaraní (22059 habitantes), ava guaraní (17592 habitantes), diaguita (14810 habitantes (entre las provincias de Salta, Jujuy, Tucumán). Este número de población originaria más otras etnias hasta totalizar la cantidad de 140.992 habitantes.

Articulando las citadas condiciones de pobreza y vulnerabilidad social en la región y en la provincia más la pluralidad étnica - cultural de su grupo poblacional se puede inferir el plausible estado de debilidad de sus condiciones para construir educación y cultura.

\section{Desigualdad social y diversidad cultural de los sujetos estudiantes}

Del contexto social recién reseñado emerge gran parte de la población estudiantil concurrente a la universidad pública y gratuita, del medio provincial. La matrícula en carreras de formación docente ha aumentado, según datos elaborados por el Departamento de Estadísticas de la Universidad Nacional de Salta (2015) pasando del medio centenar en el 2005 a un número de cien al cabo diez años. No obstante, las cifras de abandono son críticas, lo que señala problemas de deserción.

Se procede a estudiar la configuración sociocultural y de antecedentes escolares de los estudiantes inscriptos en los profesorados que ofrece la Facultad de Ciencias Exactas, dado el interés por reconocerlos como sujetos en situación de desarrollo vital y cultural en el ámbito universitario local; en especial se considera a aquéllos cuyas condiciones sociales y de diferencia étnica problematizan su adaptación a los requerimientos formales universitarios.

La aplicación de encuestas al inicio de las clases (Cátedra Introducción a la Educación, 2015, 2016) arroja información de sus antecedentes escolares, sus condiciones para el aprendizaje universitario y su medio de procedencia. Complementariamente se reconocen antecedentes escudriñando datos de orden familiar, para lo que se recurre a leer documentación correspondiente a la inscripción institucional de los sujetos que ingresan. Según los datos recogidos, estudiaron en escuelas públicas, mayormente proceden del área sub-urbana de Salta y algunos son del interior de la Provincia; entre éstos, varios pertenecen a comunidades de pueblos originarios, los más se identifican como "criollos" o mezcla de español y población local. Respecto de antecedentes de estudios de los padres, llamativamente exponen que la madre cursó el secundario, o no lo completó pero accedió al nivel. El ingreso familiar es básico o superior al básico. Los estudiantes procedentes del interior tienen menor ingreso familiar que los de la ciudad de Salta.

En las encuestas también se solicitan y registran datos de tipo sociocultural, en especial de sus intereses de lectura, entretenimientos, adhesión a grupos, formas de interrelación social. 
Se obtiene que leen en cantidad mínima y efectúan un limitado acceso a fuentes (periódico), escuchan música rock y alguna otra, no hacen bailes especiales, no escuchan conciertos sino ocasionalmente. En estos datos de los ingresantes se ha debido pensar en los últimos años, ante el reconocimiento de una baja en las disposiciones y condiciones para el aprendizaje. Asimismo, se observa en ellos la acentuación de carencias en el haber de competencias lectoras y en el dominio de la escritura, las que se juzgan reserva básica en la profesionalidad docente que se planea alcanzar u obtener. Todo demuestra la necesidad de realizar un arduo trabajo pedagógico que posibilite la construcción de aprendizajes de los nuevos sujetos ingresantes. Si a estas diversidades que caracterizan a sus identidades plurales, y que muchas veces son objeto de deslegitimación, se suman los efectos de la condición de pobreza, la combinación se torna complicada ( $c f$. Ministerio de Educación - Kaplan - García, 2006).

Se podrían seguir enumerando otras posibles relaciones entre factores de tipo social que echen luz sobre razones explicativas respecto de las transformaciones competenciales o de toma de posición sobre el valor del estudio, asumidas por los sujetos. Sin embargo, ubicado el contexto social y cultural de procedencia de los nuevos universitarios, se arrojan hipótesis específicas para fundamentar los cambios referidos, entre la matriz de estudio esperada en la universidad por parte de aquellos sujetos y sus "evasiones", o desinterés, o desencaje con las exigencias académicas.

Por un lado es real y comprobado que hay desigualdad social desde el origen en estos estudiantes, pero a la vez el área de la desigualdad se extiende e incluye a los sitios domiciliarios de procedencia, el tipo de vivienda que los aloja, el barrio en que ella se instala ( $c f$. Rosanvallon, 2007), las condiciones de las rutas, caminos o calles de acceso, el medio de transporte con que puede acceder desde la zona de residencia, y la distancia respecto al centro universitario; y también hay diferencia de disponibilidad monetaria, lo que condiciona el acceso a los materiales de estudio (fotocopias o compilados en red y en plataforma). La desigualdad ya no se define sólo por el nivel económico de ingresos o por la clase o sector social de pertenencia, que -profundamente- separa, desintegra y diferencia a los sujetos- sino se agudiza por la distinción que impone la sociedad neoliberal instalada en la región. Con ella se acentúa el cambio de condiciones laborales que conduce a la semiocupación, a la desocupación y en casos extremos a la exclusión (Castell, 2015) y demás pérdida de derechos civiles y sociales de los pobladores.

En esta metamorfosis de la cuestión social-extrapolando la cita del título del libro de Castell (1997)- los estudiantes universitarios de los profesorados de ciencias exactas demuestran efectos de la desigualdad social aludida. A ello se añade su distinción por la diferencia étnicocultural de pertenencia.

Tal distinción como "pueblos originarios" une a la población joven con sus antecesores en lazos filiatorios de sangre, pero especialmente los "enlaza" en la responsabilidad expresa o implícita de preservar sus bienes cultural-simbólicos. Pero, por fuera de las prácticas conservacionistas y de afectividad que los sujetos experimentan, la sociedad traza ideológicamente en principio- líneas divisorias de distinción y diferencia manifiestas mediante categorías de oposición binaria entre "el occidente y la región nativa", "el blanco y el originario", "lo cosmopolita y lo autóctono", "el poder y la dependencia", impregnando un juego de participación reaccionaria en la vida cívica, en el acto social y en la concurrencia 
a la escuela o a la universidad. En este último sentido, no hay que olvidar que "la diversidad es consustancial a la educación" (Rodríguez Cancio, s/f: 1083):

Todos los individuos y grupos tienen derecho a ser diferentes, a considerarse y ser considerados como tales. Sin embargo, la diversidad de las formas de vida y el derecho a la diferencia no pueden en ningún caso servir de pretexto a los prejuicios raciales; no pueden legitimar ni en derecho ni de hecho ninguna práctica discriminatoria... (Declaración de la UNESCO, 1981 en Rodríguez Cancio, s/f: 1082).

Hacia fines del siglo pasado y en lo que va del presente, refiriéndose a los colectivos descalificados, se expande el reconocimiento de "la historia de discriminación que han sufrido algunos de estos grupos sociales debido a su raza, cultura, y/o género" (Bolívar, 2005: 10).

No se dice aquí que los docentes manifiesten posesión evidente de prácticas adversas a admitir la diferencia de un buen número de sujetos estudiantes de los profesorados. Pero en los variados modos de observar la trama significativa de las conductas, emergen comentarios, apreciaciones que -quizás de modo no consciente- niegan o rechazan al otro diferente. De este modo en la vida universitaria el espacio social se fragmenta, escinde y se insensibiliza por los que son diferentes.

Según Cerletti la identidad y la diferencia implican procesos dialécticos. Expresa:

La "identidad" es caracterizada como una serie de rasgos, de deseos, de prácticas, o un conjunto abstracto de propiedades que sostienen algún tipo de invariabilidad en lo que se es. ( ). La identidad es lo que no se hace diferente, lo que en el devenir no se transforma. ( ) Por otro lado, la identidad es lo que hace diferente de lo demás, lo que diferencia frente al resto. ( ) El derecho a la diferencia es entonces, fundamentalmente, el derecho a la identidad (2013: $24-25)$.

Parada frente a estas propiedades la universidad no llega a asumirlas, sosteniendo, en cambio, una visión totalizadora, absoluta, en función de la cual llega a poner en tela de juicio las posibilidades de aprendizaje de sujetos que son los otros diferentes o diversos.

Se observa que los parámetros de interpretación y evaluación que los docentes emplean en sus prácticas se basan en criterios de homogeneidad e inalterabilidad para comprender procesos humano-sociales que difieren respecto de la "normalidad". Basado en ello, se reconcentran pensamientos y expresiones estigmatizadoras, descalificadoras y desinteresadas hacia quienes poseen códigos propios pero distintos, unido a rasgos físicos diferenciados. Ello deriva en generar "distancia entre los códigos culturales de la escuela y los cotidianos aprendidos en los diferentes niveles de socialización", así como en las "expectativas del profesor respecto al rendimiento del alumnado" (Rodríguez Cancio, s/f: 1088). 


\section{Problemas de alfabetización y de habilitación política}

Entre las propiedades del espacio social en vida de relación se cuenta el manejo de la lengua y la riqueza de su semántica, de la comunicación que fomenta, de la posibilidad que brinda de construir la escritura como código simbólico expresivo, de la interacción que promueve entre grupos, personas y a través de medios cada vez más alcanzables y manejables (en referencia a la expansión de la internet y de las redes sociales).

El aprendizaje y el manejo de la lengua se constituyen en dominios básicos de la escolaridad de los estudiantes. A ello se agrega, como principio de acción importante, la función familiar o del núcleo primario de desarrollo en cuanto a ser un ámbito de emanación, promoción y circulación de mensajes con propósito de comunicación y expresión. A partir de tales logros, la escuela tiene una base sobre la cual montar la sistematicidad de la enseñanza de la lengua, en sus variados formatos y portación.

Aplicada la evaluación de calidad en escuelas de Salta, sin embargo, no se obtienen resultados satisfactorios: "en el Noroeste (NOA) los aprendizajes [también] están por debajo del promedio nacional, con apenas un 10,6\% de los alumnos en nivel destacado y un 35,9\% en nivel bajo" (Diario Clarín, 2015).

Datos de la aplicación del Operativo Nacional de Evaluación (ONE 2013 publicados en 2015) muestran cifras de alcance para el país:

Un porcentaje importante de estos alumnos (53\%), es decir, 145.095 estudiantes del Nivel Medio pueden dar cuenta de la información global (temas, tópicos, ideas centrales, tesis, etc.) que se desprende de los textos y realizar inferencias de mediana complejidad. Identifican géneros y subgéneros discursivos, estructuras canónicas y tipologías textuales. Reconocen recursos discursivos frecuentes en textos literarios y no literarios. Localizan información explícita en posición no destacada" (Informe ONE, 2015).

El Informe del Operativo Nacional de Evaluación (ONE 2013) continúa diciendo, “el 25,7 $\%$ de los alumnos (70.541) se ubican en el Nivel de Desempeño Bajo”.

Como en la Prueba PISA 2012:

Argentina sirve como un caso ilustrativo de la desigualdad de rendimiento educativo dentro de los países de América Latina. Allí, las regiones del Noreste, el Noroeste y Cuyo se desempeñaron por debajo del promedio nacional en matemática, lectura y ciencia. (Informe sobre Calidad Educativa en América Latina de la Comisión para la Calidad de la Educación para Todos. Diario El Tribuno, 2016).

En otra fuente, el Informe continúa diciendo:

Aún dentro de las mismas regiones de Argentina, existen enormes brechas de rendimiento entre alumnos provenientes de distintos grupos socioeconómicos. En la 
ciudad de Buenos Aires, por ejemplo, la brecha entre el puntaje de los estudiantes de nivel socioeconómico más bajo y más alto constituye casi cuatro años de aprendizaje en lectura y ciencia (Diario El Tribuno, Informe sobre Calidad Educativa en América Latina. 2016).

En la línea de la posición que aquí se sostiene, al analizar las condiciones sociales se expresa:

Las diferencias de aprendizajes entre provincias argentinas son históricas: las anteriores evaluaciones hechas en el país (pruebas ONE), así como las evaluaciones internacionales, ya venían mostrando la misma brecha, que todavía no pudo cerrarse.

De acuerdo a estos resultados, vemos que el desafío más fuerte que tenemos es el de la inequidad, mucho más que el modelo de gestión. Estas pruebas siguen mostrando que los chicos pobres tienen resultados pobres. Hay que revertirlo para que todos tengan el mismo nivel (), agrega Soledad Acuña, ministra de Educación porteña (Diario Clarín, 2017).

Al llegar a la universidad los estudiantes muestran problemas recurrentes, los que no se mencionan aquí por su pertenencia al área de lengua si no se integran en el concepto de alfabetización académica (Carlino, 2005). La autora entiende por tal "el conjunto de nociones y estrategias necesarias para participar en la cultura discursiva de las disciplinas, así como en las actividades de producción y análisis de texto requeridas para aprender en la universidad" (2005: p. 5).

Ello promueve que los estudiantes participen en las prácticas de lectura, escritura y pensamiento propias de cada campo de estudio, con una fuerte participación docente desde una concepción no centralista sino colaboradora de su proceso de elaboración cognitiva y de aprendizaje.

En especial pensando en los problemas de dominio de las mencionadas prácticas por la pertenencia a sectores sociales con pobreza lingüística, con escasa práctica de la escritura o con acceso a cortos mensajes a través de las redes sociales, y porque hay escasez de capital cultural familiar en el núcleo de amistades o en las prácticas del entorno social inmediato, se agudiza el análisis del proceso de pensamiento al que el sujeto se remite para interpretar lo leído, organizar la escritura, articular competencias entre la lectura, la escritura y la crítica al contenido. Se destaca, a nivel de aula universitaria, la necesidad de superar la lectura mecánica, saber localizar textos de información (a nivel de educación superior), distinguir la trama organizacional básica de un texto, diferenciar las clases de palabras usadas en un texto o reconocer el nuevo vocabulario e incorporar en el haber lingüístico-cognitivo, captar la secuencia argumentativa y diferenciar los nodos importantes cualificándolos $\mathrm{o}$ categorizándolos. Desde la actuación profesional docente convendrá seleccionar textos para su lectura e interpretación que sean inicialmente "similares en tipología, complejidad, extensión o densidad textual" [y poco a poco irán complejizándose] (Informe de Resultados ONE-Censo: 17).

Según la misma fuente, un Nivel Medio o Alto de Desempeño incluye "leer con estrategias de relectura total y parcial, diferenciar, cotejar e integrar información, construir significados 
globales a partir de la ratificación y/o rectificación de hipótesis de lectura y realizar inferencias de distinto grado de dificultad" (Informe de Resultados ONE-Censo: 17).

A este arduo trabajo de alfabetización académica se articula el desarrollo de la habilitación política en función de lo cual los sujetos aprenden a leer lo connotado en el texto, a descifrar el propósito ideológico o la intencionalidad del mensaje que se quiere transmitir o se invita a adherir, y la sutileza de la construcción de grandes relatos (al modo como lo expresa Lyotard) que buscan generar credibilidad. Armar hipótesis sobre ideas procedentes de enfoques contrapuestos e ideologizados sería también un camino para invitar, pedagógicamente, a construir la respuesta.

No obstante, la tarea, no siendo fácil, no resulta imposible. El trabajo que se desarrolla en la cátedra de primer año es arduo partiendo de las condiciones de limitaciones culturales y sociales de los estudiantes; la tarea en equipo permite acompañar y brindar asesoramiento constante y sostenido de modo que, hacia el mes de iniciado el cuatrimestre, se observan logros en su cuantía y calidad expresiva, en el desarrollo de destrezas de lectura, en la construcción lógica de las ideas nodales de los textos de lectura. Los estudiantes van logrando construir sus aprendizajes y los profesores van trabajando su habilitación para la alfabetización académica y la lectura política del hecho educacional.

Como dice Geneyro (2007: p. 6) en su pronunciamiento que aún hoy se mantiene:

Lograr una universidad más inclusiva no es un desafío menor, a la par que genera más de un dilema. Uno de ellos consiste en cómo incluir y cómo retener a quienes eligen cursar estudios universitarios, máxime cuando muchos aspirantes provienen de poblaciones con escasa posesión de bienes culturales y desfavorables condiciones socioeconómicas, sin que ello conlleve desmerecer la calidad de la educación que se brinda.

\section{Líneas de políticas y estrategias de educación}

Según se alude en el trabajo la configuración del estudiantado concurrente a carreras de formación docente en el área de ciencias exactas cambia en las últimas décadas, observándose deficiencias en su capital de habilitación para el estudio universitario (lectura, escritura, organización de tareas) que tornan difícil su alfabetización académica. De allí la importancia de fortalecer, comprometer y asegurar la tarea pedagógica de los docentes.

La tarea es decididamente política dado que implica la preparación de futuros docentes de un tiempo social con nuevos requerimientos técnicos de manejo, pero también con exigencias de planteamiento y organización de criterios de razonabilidad, sensibilidad y justicia social para trabajar con estudiantes de escuelas públicas. Es probable que los estudiantes que hoy pueblan las aulas universitarias vivenciarán una sociedad dual, en una región dependiente y con fragilidad -y vulnerabilidad- en sus derechos y condiciones de vida.

Porque a la vez ésta es la configuración política del Estado que se constituye en el país y la región en la actualidad. 
Para contribuir a contrarrestar el restablecimiento o el logro de condiciones de igualdad de oportunidades desde el inicio de los estudios universitarios, de reconocimiento de sus diferencias identificativas, étnicas, de clase y de género, y para favorecer un trabajo basado en la justicia social en el orden académico, se propician las siguientes líneas de acción y estrategias o dispositivos consecuentes:

-En cuanto a establecerse como acción y actividad universitaria específica en la Universidad Nacional de Salta:

- Acentuar la centralidad de la visión política en tiempos en que el mercado y los intereses financieros comprimen la acción estatal redistributiva, mejorando la direccionalidad social de la universidad pública.

. Asegurar los principios legales que la definen como institución superior sostenida por el Estado, según la norma de Implementación efectiva de la Responsabilidad del Estado en el nivel de Educación Superior, en tanto "la educación y el conocimiento son un bien público y un derecho humano y personal (art. 2), garantizando la igualdad y condiciones de acceso, la permanencia, la graduación (art. 2 inc. a), [así como] proveer equitativamente becas, infraestructura adecuada y recursos tecnológicos (art. 2, inc. b) para los sectores carentes, promover políticas de inclusión educativa (art. 2, inc. c) en relación con la diferencia de género, cultural-étnica" (Ley 27204/2015).

En definitiva, remite al accionar de los Consejos de gobierno universitario defendiendo la educación pública, gratuita y de amplio ingreso.

-En el orden de las políticas académicas:

- Evaluar las experiencias de ingreso amplio o irrestricto, considerando la necesidad de brindar un real servicio de resguardo del derecho a la educación de la población juvenil más desfavorecida, para aplicar una política de distribución social de las oportunidades educativas.

. Organizar el acceso a datos de matrícula que, al interpretarse, no estigmatice a la población juvenil mencionada sino se elabore con neutralidad profesional.

-En el orden de las políticas de la Facultad de Ciencias Exactas:

. Establecer un Observatorio de Información sobre el Avance Curricular de los estudiantes de los Profesorados, cuyo análisis profesional comunique -para fundamentar la toma de decisiones- avances y obstáculos curriculares de los estudiantes, con vistas a su superación.

. Continuar la política de tratamiento del ingreso y la prestación de servicios de atención al ingresante (tutorías, orientación pedagógica...) bajo una constante evaluación crítica en cuanto a los marcos teóricos de manejo y de respaldo al abordaje profesional docente. 
. Propiciar y fomentar seminarios, talleres, cursos de postgrado que permitan revisar el formato homogéneo del aula universitaria, para poder situar y asumir la política de la diferencia y la diversidad, afín al derecho a la educación con dignidad, sin segmentación, sub-alteridad ni exclusión.

. Entre estas actividades se sugiere particularizar el montaje de seminarios o foros sobre: alfabetización académica (Carlino, 2005), comprensión e interpretación de textos y su fundamento psico-educacional

. Profundizar la discusión sobre posibles cambios de formatos de clases que rompan la unidireccionalidad de la voz docente y se reemplace por la relación compartida con los estudiantes, coordinados en la construcción de sus aprendizajes.

-A nivel de aula universitaria:

. Fomentar la provisión gratuita y accesible de lecturas en variados soportes y a través de distintas vías de acceso y comunicación entre estudiantes y estudiantes - docentes.

. Discutir los modos y formatos de evaluación, buscando coherencia con los planteos y estrategias de intervención en las clases. Respetar -en la enseñanza, en el aprendizaje y en la evaluación- los principios de atención a la diferencia y a la diversidad.

. Fomentar el inicio de talleres pedagógicos en escuelas de nivel medio, en sus variados formatos y en su dispersión geográfico-poblacional (escuela en contexto privado de libertad, escuela domiciliaria, escuela rural con servicio mixturado entre la educación a distancia y la presencialidad; en la ciudad, en villas y barriadas) fomentando el respecto a la diferencia, igualdad y equidad.

-A nivel de fomento y expansión cultural de los estudiantes, asumiendo la producción cultural no por su selectividad ("alta cultura") sino por su valor semántico-significativo, expresivo, del hombre y su realidad. (cf. Geertz, 1987):

. Promoción de videos, documentales, cortos cinematográficos, música a difundir en espacios de tránsito común en la Facultad. Idénticamente promoción de obras de arte, ciencia, técnica (en vivo o reproducciones) por períodos secuenciales.

. Organización de visitas, charlas y debate con personalidades de la cultura, del periodismo, de los medios de comunicación, de dirigentes de centros vecinales, de ciudadanos de pueblos originarios, cubriendo un calendario trazado.

Se trata de trabajar un programa de formación docente de futuros profesores en ciencias exactas, cuya excelencia académica está garantizada en este medio universitario, pero que además les provea de la habilitación política que posibilite -en su presente de estudiantes y en su futuro profesional- asegurar derechos de educación para la inclusión de la amplia franja poblacional pluriétnica, pluriclasista e identitaria, a quienes enseñarán. 


\section{Conclusiones}

Basado en el interés por el reconocimiento de estudiantes que ingresan a las carreras de formación docente en la Facultad de Ciencias Exactas de la Universidad Nacional de Salta, el trabajo se preocupa en especial por la situación de carencias de tipo cultural social incidentes en problemas de rendimiento académico, de sujetos identificados por su carácter diferencial en tanto pertenecen a etnias originarias o a ambientes de escasa promoción social y cultural.

Se hipotetiza que la universidad no ha efectuado aún un cambio de posición -ni ideológica ni epistemológica- respecto de la aceptación efectiva del ingreso de estos estudiantes que deberían poder elaborar un proceso de alfabetización académica promovido y acompañado por docentes que busquen aseguran su ingreso y avance curricular.

Se emplean fuentes de documentación y bibliografía atinente para refrendar lo expuesto.

Se finaliza trazando líneas de políticas y estrategias de orden institucional, políticoeducativo, instrumental y de políticas académicas que favorezcan la concreción de trabajar en pos de la "identidad, igualdad y educación”, como afirma Cerletti (2013).

\section{Referencias}

Baudelot, Christian, y Establet Roger (1976) La escuela capitalista en Francia. Madrid. S XXI Ed., 2da. Edición.

Bourdieu, Pierre (1977) La reproducción. Elementos para una teoría del sistema de enseñanza. Barcelona: Editorial Laia.

Bowles, Samuel, Gintis, Hebert (1985) La instrucción escolar en la América Capitalista; la reforma educativa y las contradicciones de la vida económica. Madrid. S XXI Ed.

Bruner, Jerome (2000). La educación, puerta de la cultura. Madrid: Visor, Col. Aprendizaje.

Bolívar, Antonio (2005) Equidad educativa y teorías de la justicia. REICE. Revista Iberoamericana sobre Calidad, Eficacia y Cambio en Educación, vol. 3, num.2, pp. 42-69. Recuperado de https://bit.ly/2t7EzBw

Cátedra Introducción a la Educación. Encuesta Diagnóstico inicial. Facultad de Ciencias Exactas - Universidad Nacional de Salta, 2015.

Cátedra Introducción a la Educación. Encuesta Diagnóstico inicial. Facultad de Ciencias Exactas - Universidad Nacional de Salta, 2016.

Carlino, Paula. (2005). Escribir, leer y aprender en la universidad. Una introducción a la alfabetización académica. Buenos Aires: Fondo de Cultura Económica. pdf. 45 p. 
Castel, Robert. (2015) Las trampas de la exclusión. Trabajo y utilidad social. Ed. Topía. Colección Fichas para el S XXI.

Castel, Robert (1997) La metamorfosis de la cuestión social: una crónica del salariado. Paidós Ed.

Cerletti, Alejandro (2013) Identidad, igualdad y educación. En Revista Praxis y Saber. Revista de Investigación y Pedagogía. Vol. 4. Núm. 7 - Enero - Junio 2013 - Pág. 1733.

Geertz, Clifford 1987) La interpretación de las culturas, México, Editorial GEDISA.

Colque, Valeriano, economista (2017) -Nota: "Lo más preocupante no es la pobreza sino la desigualdad". Recuperado de https://bit.ly/2ybvYnm

Diario Clarin (2015) Los estudiantes del norte del país aprenden menos que los demás, Recuperado de https://clar.in/2169DhD

Diario Clarín (2017) Brecha educativa: los estudiantes del norte del país aprenden menos que los demás. Recuperado de https://clar.in/2LPdcE6

Diario El Tribuno - Salta (2016) Estudiantes del NOA, por debajo del rendimiento promedio del país. Recuperado de https://bit.ly/2Jz3RDL

Instituto de Estudios sobre Estado y Participación - IDEP. ATE - Asociación de Trabajadores del Estado Informe Provincia de Salta -, Salta (2008). pdf. Recuperado de https://bit.ly/2sVPajL

Instituto de Estudios Laborales y del Desarrollo Económico-IELDE. Pobreza en Salta. pdf. Recuperado de https://bit.ly/2JT8Dvc.

Geneyro, Juan Carlos (2007) Algunos dilemas y desafíos para la Universidad. En Salud Colectiva. Buenos Aires, 3 (1): 5 -7. Enero-Abril. pdf. Recuperado de $\underline{\text { https://bit.ly/2HJusbD }}$

Gvirtz S., Grinberg S., Abregú V. (2009) La educación ayer, hoy y mañana. El ABC de la Pedagogía. Buenos Aires: Aique Grupo Editor. Cap. 1.

Informe Ejecutivo CENSO ONE 2013 - CENSO 5\% $6^{\circ}$ Año. Recuperado de https://bit.ly/2lgWeTO.

Ley 27204/2015 de Implementación efectiva de la Responsabilidad del Estado en el nivel de Educación Superior. Boletín Oficial de la República Argentina. Recuperado de https://bit.ly/2JMO1FS. 
Ministerio de Educación, Ciencia y Tecnología (Argentina) - Kaplan, Carina - García, Sebastián (2006) La inclusión como posibilidad. Recuperado de https://bit.ly/2K4y4u9.

Rodríguez Cancio, Mónica (s/f) Concepto de diversidad. Recuperado de https://bit.ly/2ylSZUI.

Rosanvallon, Pierre (2007) La nueva cuestión social. Repensar el Estado providencia. Buenos Aires: Manantial.

Tenti Fanfani, Emilio (Compilador) (2003) Educación Media para todos. Los desafíos de la democratización del acceso. Fundación OSDE, Unesco - IIPE, Grupo Editor Altamira.

Universidad Nacional de Salta - Departamento de Estadística (2015) Informe de Desgranamiento de Alumnos a 2015, según año de ingreso. 